\title{
SMR
}

\section{Suppression of lentivirus-mediated transgenic dendritic cells in graft-versus-host disease after allogeneic bone marrow transplantation in mice}

\author{
Y.J. Xu${ }^{1 *}$, W.R. Chen ${ }^{1,2 *}$, D.P. Li ${ }^{1}$, L.X. Song ${ }^{1}$, J.Q. Wu ${ }^{2}$, P. Zhang ${ }^{1}$, \\ Z.Y. Li $^{1}$ and Y.H. Huang ${ }^{1,2}$ \\ ${ }^{1}$ Department of Hematology, The Affiliated Hospital of Xuzhou Medical College, \\ Xuzhou, Jiangsu, China \\ ${ }^{2}$ Department of Hematology, \\ The Third Affiliated Hospital of Xuzhou Medical College, Xuzhou, Jiangsu, \\ China \\ *These authors contributed equally to this study. \\ Corresponding author: Y.H. Huang \\ E-mail: hxr1583@sina.com / hongyihong_1@163.com
}

Genet. Mol. Res. 14 (3): 11444-11455 (2015)

Received February 15, 2015

Accepted May 15, 2015

Published September 25, 2015

DOI http://dx.doi.org/10.4238/2015.September.25.11

\begin{abstract}
We determined whether genetically engineered immature dendritic cells (imDCs) mediated by lentiviral vectors alleviate acute graft-versus-host disease (GVHD) after allogeneic bone marrow transplantation (allo-BMT) in mice. We introduced the mouse chemokine receptor $7(\mathrm{Ccr} 7)$ gene into the bone marrowderived imDCs of C57BL/6 mice to construct genetically engineered imDCs. A 1:1 mixture of bone marrow and spleen cells from the donors was injected into the recipients, which were divided into four groups: radiation, transplantation, empty vector, and transgenic imDC groups. Symptoms, clinical scores, GVHD pathological changes, and survival times and rates of recipients were recorded; secretion of IFN- $\gamma$ and IL-
\end{abstract}


4, and allogeneic chimerism rates were detected. The survival time of the transgenic imDC group ( $27.5 \pm 7.55$ days $)$ was significantly longer than in the other three groups $(\mathrm{P}<0.01)$. The GVHD score of the imDC group mice was significantly lower than in the transplantation and empty vector groups $(\mathrm{P}<0.05)$, which meant that mice in the transgenic imDC group had the lightest pathology damage in the target organs. In the transplantation group, IFN- $\gamma$ increased while IL-4 decreased. In contrast, IFN- $\gamma$ decreased and IL-4 increased in both empty vector and trans-imDC groups, and the difference was significant in the latter $(\mathrm{P}$ $<0.01)$. Thirty days or more following transplantation, the allogeneic chimerism rate was still $95-100 \%$, suggesting complete donor type implantation. $\mathrm{Ccr} 7$ transfection into imDCs suppressed occurrence and severity of acute GVHD after allo-BMT in mice; the mechanism might be associated with IFN- $\gamma$ decrease and IL-4 increase.

Key words: Dendritic cells; Recombinant lentivirus; Transgenic; Chemokine; Immune tolerance; Graft-versus-host reaction

\section{INTRODUCTION}

Allogeneic hematopoietic stem cell transplantation (allo-HSCT) is a major therapeutic method in the treatment of high-risk hematologic malignancies and many other life-threatening genetic diseases. However, as one of the complications of allo-HSCT, graft-versus-host disease (GVHD) seriously affects a patient's prognosis and quality of life (Choi et al., 2010; Travnik et al., 2011), making the prevention of acute GVHD the key to successful allo-HSCT therapy. Removal of T cells before transplantation and the application of immunosuppressants are the primary strategies in the prevention and treatment of GVHD, yet the former increases the incidence of graft rejection and leukemia relapse, while the latter has severe toxic side effects that often lead to lethal infections. Studies have demonstrated that an immune tolerance of the graft by the host induced by adoptive immunotherapy of mesenchymal stem cells, (Edinger et al., 2009; Huang et al., 2010; Alpdogan and van den Brink, 2012), regulatory T cells, natural killer cells, dendritic cells, etc., has shown favorable results in the prevention and treatment of GVHD (Cools et al., 2007; Joo et al., 2010; Morales-Tirado et al., 2011). Dendritic cells (DCs) comprise heterogeneous cell populations with different subsets and various functions. As the most powerful antigen-presenting cells (APCs), DCs have a dual immunological function that can either activate or suppress immune responses depending on their maturity and local microenvironment (Jin et al., 2004; Hopkins and Connolly, 2012). It is currently acknowledged that DCs play a pivotal role in the development of immune tolerance. Immature DC (imDC)-mediated specific immune tolerance has received increased attention (Ueno et al., 2007; Zhou et al., 2013). Recent studies have revealed that imDCs play an important role in the induction of immune tolerance by migrating to secondary lymphoid organs, and stimulate $\mathrm{T}$ cells to generate regulatory T cells (Wilson et al., 2003; Cools et al., 2008).

It has been proved that chemokines are important in the migration, differentiation, maturation, and antigen-presenting function of DCs in vivo. The chemokine CC motif receptor 7 (CCR7) was first found to be expressed on the surface of immune cells such as naive $T$ cells, B cells, and DCs in research on the Epstein-Barr virus infection of B cells. Subsequent 
research found that CCR7 is upregulated during the maturation process of DCs and plays a crucial role in the chemotactic migration of DCs from peripheral tissues to secondary lymphoid organs after binding with its ligands CCL21 and CCL19 (Pan et al., 2008). In another study, it was shown that bone marrow-derived DCs did not migrate to the lymph nodes in $\mathrm{Ccr} 7$ gene knockout ( $\left.\mathrm{Ccr}^{-/}\right)$mice (Hintzen et al., 2006). However, since imDCs lack CCR7 expression (Xin et al., 2009), their ability to migrate to the lymph nodes is weaker, which causes them to develop into mature DCs with little inhibition effect on T lymphocyte proliferation during their long stay in non-T cell zones. Therefore, by constructing modified imDCs that express chemokine receptors on their surface, which have a strong homing capacity for the draining and central lymph nodes, there is a great probability that their ability to induce immune tolerance may be enhanced. In early experiments, our research team successfully constructed lentivirus vectors carrying the mouse $\mathrm{Ccr} 7$ gene (labeled with green fluorescence), namely, LV-CCR7, and obtained imDCs with high expression of CCR7 by infecting imDCs with the above-mentioned virus vector (Xin et al., 2009; Cao et al., 2010). Therefore, the aim of our present study was to investigate the impact of $C$ cr 7 -modified imDCs on GVHD after allo-HSCT and the mechanisms involved, so as to provide evidence for the prevention and treatment of GVHD in clinical practice.

\section{MATERIAL AND METHODS}

\section{Animals}

$\mathrm{C} 57 \mathrm{BL} / 6$ mice $\left(\mathrm{H}-2^{\mathrm{b}}\right)$ were the donors and $\mathrm{BABL} / \mathrm{c}$ mice $\left(\mathrm{H}-2^{\mathrm{d}}\right)$ were the recipients. All mice were male, 18-22 g, and 8-12 weeks old; they were bought from the Experimental Animal Center of Yangzhou University and raised in the SPF animal room of the Experimental Animal Center of Xuzhou Medical College. The cages were sterilized in 1:10,000 dilution chlorine and changed once per day. Fodder and sawdust bedding were sterilized by high pressure steaming, and drinking water was sterilized by boiling. Erythromycin $(250 \mathrm{mg} / \mathrm{L})$ and gentamicin $(320 \mathrm{mg} / \mathrm{L})$ were added to the drinking water one day before the transplantation. All dispositions during the experiments complied with the animal ethics standards.

\section{Reagents}

Mouse GM-CSF and IL-4 were purchased from the PeproTech, Inc., Rocky Hill, NJ, USA; Roswell Park Memorial Institute (RPMI) 1640 medium, fetal bovine serum, and Dulbecco's modified Eagle's medium were purchased from the Gibco-BRL, Invitrogen Life Technologies, Carlsbad, CA, USA; Mouse IL-4 and IFN- $\gamma$ enzyme-linked immunosorbent assay kits were purchased from the Shanghai JITAI Biotechnology Company, China; FITC-H-2K ${ }^{\mathrm{d}}$ and PE-H-2K $\mathrm{K}^{\mathrm{b}}$ (FITC is fluorescein isothiocyanate and PE is phycoerythrin) were purchased from the BD Pharmingen, San Diego, CA, USA; FITC-labeled anti-mouse CD11c antibody, FITC-IA/IE antibody, PE-labeled CCR7, CD80, and CD86 antibodies and the corresponding isotype control antibodies were purchased from the eBioscience San Diego, CA, USA; Exodus-2 (CCL21) antibody was also bought from the PeproTech, Inc. The lentivirus that carried the $\mathrm{Ccr} 7$ gene and green fluorescent protein were constructed and preserved in our laboratory (Xin et al., 2009; Cao et al., 2010). DCs infected by the target gene-carrying virus and the empty virus were designated as CCR7-imDCs and pXZ9-imDCs, respectively. Mitomycin 
$\mathrm{C}$ and 3-(4,5-dimethylthiazol-2-yl)-2,5-diphenyltetrazolium bromide were purchased from Sigma-Aldrich Inc., Seelze, Germany, and a Transwell chamber was bought from the Corning Costar Corporation, USA.

\section{Extraction of bone marrow and spleen cells from donor mice}

C57BL/6 mice were sacrificed by cervical dislocation, and bone marrow in the femur and tibia was taken under sterile conditions and made into a mononuclear cell suspension, which was adjusted to a density of $5 \times 10^{7}$ cells $/ \mathrm{mL}$ in RPMI 1640 medium. The spleen tissue from the C57BL/6 mice was taken and made into a spleen cell suspension, which was then separated using lymphocyte separation medium into mononuclear cells and adjusted to a density of $5 \times 10^{7}$ cells $/ \mathrm{mL}$. Cell viability of more than $95 \%$ was confirmed by trypan blue staining.

\section{Culture, transfection, and function test of mouse imDCs}

A bone marrow cell suspension from the $\mathrm{C} 57 \mathrm{BL} / 6$ mice was collected by the routine method in our lab and seeded on six-well plates at $1 \times 10^{6}$ cells per well. Then mouse imDCs were prepared by referring to the method described in the literature (Xin et al., 2009; Wang et al., 2012). The virus carrying the $\operatorname{Ccr} 7$ gene (pXZ9-Ccr7) and the empty virus control (pXZ9) were added to the different groups of imDCs for transfection, and the corresponding transfected imDCs were designated CCR7-imDC and pXZ9-imDC, respectively. After transfection, a large amount of enhanced green fluorescent protein expression (in about $50 \%$ of cells) could be seen in the CCR7-imDC group under a fluorescence microscope. Flow cytometry revealed no expression of CCR7 in the pXZ9-imDC group, while there was CCR7 expression in the CCR7-imDC group. Moreover the expression level of CCR7 was higher in the immature DC group than in the mature DC group, and the expression of CD80, CD86, CD11c, and MHC- II molecules was quite low in immature DCs transfected with the $C c r 7$ gene, suggesting that $C c r 7$ had been successfully introduced into the imDCs, which led to effective CCR7 protein expression (Xin et al., 2009). The 3-(4,5-dimethylthiazol-2-yl)-2,5-diphenyltetrazolium bromide assay was used to detect the impact of DCs on T cell proliferation in each group, and Transwell chamber culture was used to detect the in vitro chemotaxis of cells in each group.

\section{Establishing the mouse GVHD model}

As recipients, the $\mathrm{BABL} / \mathrm{c}$ mice received total body irradiation (TBI) using an X-ray linear accelerator at a total dose of $7.5 \mathrm{~Gy}$ (dose rate $0.67 \mathrm{~Gy} / \mathrm{min}$ for $4 \mathrm{~h}$ ) before transplantation on the same day. The recipient mice were divided by random number table into the following four groups (10 in each group, mice that were sacrificed for chimera analysis and cytokines detection were not included): 1) simple TBI group, received an infusion of $0.3 \mathrm{~mL}$ RPMI 1640 medium via the tail vein; 2) allo-BMT group, received an injection of $5 \times 10^{6}$ bone marrow cells $+5 \times 10^{5}$ spleen cells from donor mice via the tail vein; 3) pXZ9-imDC group, received an injection of $5 \times 10^{6}$ bone marrow cells $+5 \times 10^{5}$ spleen cells from donor mice + $5 \times 10^{6} \mathrm{pXZ9-imDC}$ (empty vector transduced imDCs) via the tail vein; and 4) CCR7-imDC group, received an injection of $5 \times 10^{6}$ bone marrow cells $+5 \times 10^{5}$ spleen cells from donor mice $+5 \times 10^{6} \mathrm{CCR} 7-\mathrm{imDC}$ vial the tail vein. 


\section{Outcome measurement after transplantation}

The body weight of the recipient mice was measured regularly, and feeding and mental conditions were observed and recorded every day. The survival times and rates of the recipients were also recorded and calculated. Peripheral blood counts and blood smears were performed regularly to observe hematopoietic recovery: white blood cells (WBCs) $<0.5 \mathrm{x}$ $10^{9} / \mathrm{L}$ for graft failure and $\mathrm{WBC}>1.0 \times 10^{9} / \mathrm{L}$ for hematopoietic recovery. Criteria for acute GVHD included peripheral WBC $>1 \times 10^{9} / \mathrm{L}$ accompanied by fatigue, poor activity, weight loss, arching, curling hair, diarrhea, and other symptoms. Severity of GVHD was evaluated by the integral system reported by Cooke et al. (1996), which comprised the following five indicators: weight loss, poor activity, body posture, curling hair, and skin ulcers, totaling 10 points. Except for deaths caused directly by GVHD, any death caused by hematopoietic suppression (infection, bleeding, and other conditions) within two weeks was defined as transplant-related.

\section{Pathological observation of GVHD target tissues}

In each group, target tissues (plantar skin, liver, and small intestine) of the moribund mice and mice that were sacrificed after 30 days were fixed with $100 \mathrm{~g} / \mathrm{L}$ formaldehyde solution and made into paraffin-embedded sections. Then the sections were subjected to hematoxylin-eosin staining to identify pathological changes of GVHD under a light microscope. Acute GVHD histological scores were calculated according to the relevant literature (Blazar et al., 1998; Kaplan et al., 2004).

\section{Determination of plasma cytokines after transplantation}

Eyeball blood from the recipient mice in the three groups (transplantation, pXZ9imDC, and CCR7-imDC groups) was taken at different time points $(0,5,10,15,20$, and 30 days after transplantation) and centrifuged to obtain blood plasma. Concentrations of IFN- $\gamma$ and IL-4 in the blood plasma were determined by enzyme-linked immunosorbent assay.

\section{Detection of bone marrow cell chimeras}

We randomly extracted $1 \times 10^{6}$ bone marrow cells from the living recipient mice 14 and 30 days after transplantation. We added PE-labeled anti-H- $2 \mathrm{~K}^{\mathrm{b}}$ monoclonal antibody into the cell suspension and incubated for $20 \mathrm{~min}$ in the dark. Hemolysin was added for $5 \mathrm{~min}$ for dissolution of red blood cells and washed away using phosphate-buffered saline. The percentage of donor mice-derived cells in each recipient mouse was detected by flow cytometry and the allogeneic chimerism rate was calculated using the CellQuest software.

\section{Statistical analysis}

Data were processed with the SPSS16.0 software and are reported as means \pm SD. Comparisons among the groups were analyzed with one-way analysis of variance, pairwise comparisons among groups were analyzed by the Q-test, and survival rates in the different groups were compared using the Kaplan-Meier method. Differences with P values of $<0.05$ were considered to be statistically significant. 


\section{RESULTS}

\section{Impact of imDCs on $\mathrm{T}$ cell proliferation}

The $\mathrm{T}$ cell proliferation rate after pXZ9-imDC stimulation was $319.96 \pm 11.51 \%$, which was quite low. In contrast, the T cell proliferation rate after stimulation by mature DCs was $361.02 \pm 14.38 \%$, which was significantly higher $(\mathrm{P}<0.05)$. The stimulatory capacity of CCR7-imDCs on T cells was lower than mature DCs, with a cell proliferation rate of $324.82 \pm$ $6.44 \%$, which was not statistically significant when compared with pXZ9-imDCs $(\mathrm{P}>0.05)$. The above results imply that the in vitro stimulation effect of $C$ cr 7 -modified imDCs on allogeneic T lymphocytes remained weak.

\section{Analysis on DC migration}

From the results of the in vitro migration assay, it could be seen that an upregulation of $C c r 7$ gene expression by imDCs enhanced the chemotaxis capacity of DCs. In the pXZ9imDC group, the in vitro cell mobility rate was $7.97 \pm 0.46 \%$, in the CCR7-imDC group it was $26.71 \pm 0.28 \%$, while cell mobility in the mature DC group was $15.88 \pm 0.43 \%$. In summary, there was a significant difference between the three groups $(\mathrm{P}<0.05)$.

\section{Survival of mice after transplantation}

Mice in the TBI group all suffered progressive weight loss and began to die on the sixth day after transplantation; all died of bone marrow failure within 11 days. The average survival time (AST) was $8.20 \pm 1.48$ days. Mice in the allo-BMT group began to show clinical manifestations of acute GVHD, such as poor feeding, weight loss, arching, curling and loss of hair, diarrhea, etc. The first death was on the fifth day after transplantation, and by the 17th day all the mice had died; the AST was $12.20 \pm 2.78$ days. Most of the mice in the pXZ9-imDC group also showed manifestations of GVHD and began to die from the 13th day, with the majority dying by the 22 nd day. However, about $10 \%$ of the mice in this group survived for more than 30 days, and the AST was $20.70 \pm 6.01$ days, which was prolonged significantly compared with the allo-BMT group $(\mathrm{P}<0.001)$. Finally, mice in the CCR7-imDC group began to die on the 15th day and $40 \%$ survived longer than 30 days, making the AST of this group 27.5 \pm 7.55 days, which was significantly longer than the AST of the allo-BMT or pXZ9-imDC groups $(\mathrm{P}<0.01)$. The results are shown in Figure 1.

\section{Severity of GVHD in each group}

Mice in the allo-BMT, pXZ9-imDC, and CCR7-imDC groups all showed different degrees of GVHD manifestations including fatigue, poor activity, weight loss, arching, curling and loss of hair, diarrhea, etc. Among the three groups, mice in the allo-BMT group suffered the most severe symptoms and were given a score of $6.90 \pm 1.66$ points. The GVHD score of the pXZ9-imDC group was $5.60 \pm 0.97$ points, while mice in the CCR7-imDC group, which had the mildest symptoms, were given $4.10 \pm 1.79$ points. In summary, among the $\mathrm{pXZ9-imDC,} \mathrm{CCR7-}$ imDC, and allo-BMT groups, the difference was statistically significant $(\mathrm{P}<0.05)$. Moreover, the difference between the pXZ9-imDC and CCR7-imDC groups was also significant $(\mathrm{P}<0.05)$. 


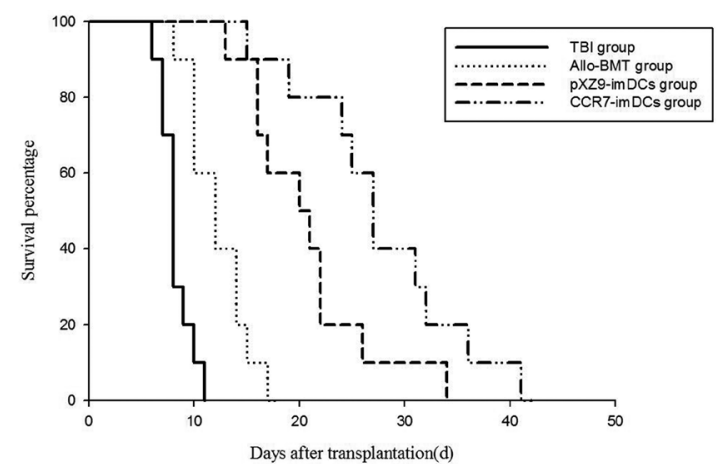

Figure 1. Post-bone marrow transplantation (BMT) survival curves for mice in the different transplantation groups.

\section{Pathological changes}

After transplantation, the pathological manifestations in the livers, small intestines, and skins of mice in each group were consistent with their clinical manifestations. In the alloBMT and pXZ9-imDC groups, there was obvious derangement of liver cells, focal necrosis, lymphocyte infiltration in the periportal area, destruction and collapse of the small bile duct in liver tissues, small intestinal epithelial cell necrosis and partial defect excalation, inflammatory cell infiltration in the underlayer, intestinal villous atrophy degeneration, and even severe structural damage to the small intestine; and in the skin tissues there was discontinuity in the skin basal layer, infiltration of lymphocytes and monocytes, and vacuolar degeneration of epithelial cells. In contrast, pathology showed only mild or no GVHD manifestations in the CCR7-imDC group (Figure 2A-F). When GVHD pathology score points were obtained according to the pathology scoring system, it was found that the pathological score was lower in the CCR7-imDC group than in the control group $(\mathrm{P}<0.05)$, while the difference was not statistically significant between the pXZ9-imDC group and the control group $(\mathrm{P}>0.05)$ (see Table 1).

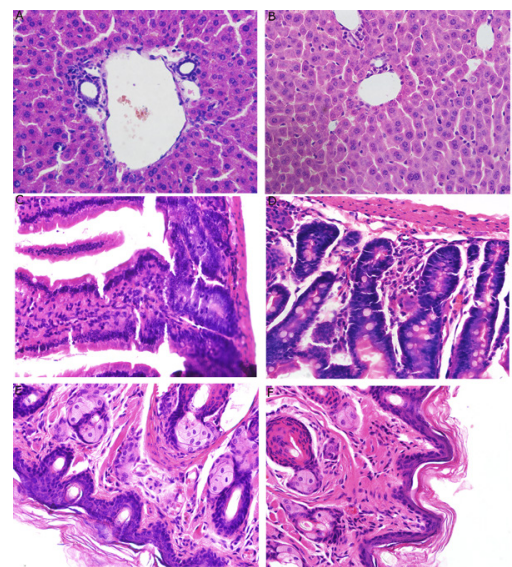

Figure 2. Histopathological evidence from liver, small intestine, and skin in mice after transplantation (hematoxylineosin-stained, magnification 400X). (A. and B. liver; C. and D. intestine; E. and F. skin). (A, C, and E. pXZ9-imDC group; B, D, and F: CCR7-imDC group). 
Table 1. Graft-versus-host disease (GVHD) pathologic scoring for liver, small intestine, and skin in mice following transplantation $(\mathrm{N}=6$, means $\pm \mathrm{SD})$.

\begin{tabular}{lccc}
\hline Group & Liver & Small intestine & Skin \\
\hline allo-BMT & $4.8 \pm 0.8$ & $5.2 \pm 0.8$ & $6.0 \pm 0.6$ \\
pXZ9-imDC & $4.3 \pm 0.5$ & $5.5 \pm 0.5$ & $5.5 \pm 0.5$ \\
CCR7-imDC & $2.3 \pm 0.5^{\sharp}$ & $2.5 \pm 0.5^{\sharp}$ & $3.0 \pm 0.6^{\#}$ \\
\hline
\end{tabular}

$\mathrm{BMT}=$ bone marrow transplantation; imDCs $=$ immature dendritic cells; $\mathrm{CCR} 7=\mathrm{CC}$ motif receptor $7 .{ }^{\#} \mathrm{P}<0.05$ vs allo-BMT group.

\section{Changes in the plasma cytokine levels of the recipients after transplantation}

In the allo-BMT group, the plasma IFN- $\gamma$ concentration kept rising and peaked on the 10th day after transplantation, while the level of IFN- $\gamma$ decreased in both pXZ9-imDC and CCR7-imDC groups and reached its lowest point on the 20th day, which was particularly noticeable in the CCR7-imDC group $(\mathrm{P}<0.01)$. Simultaneously, the plasma concentration of IL-4 decreased in the allo-BMT group and increased in both the pXZ9-imDC and CCR7imDC groups, where it peaked on the 10th day. Probably the increase was more significant in the CCR7-imDC group $(\mathrm{P}<0.01)$. The results are presented in Figure 3A and B.
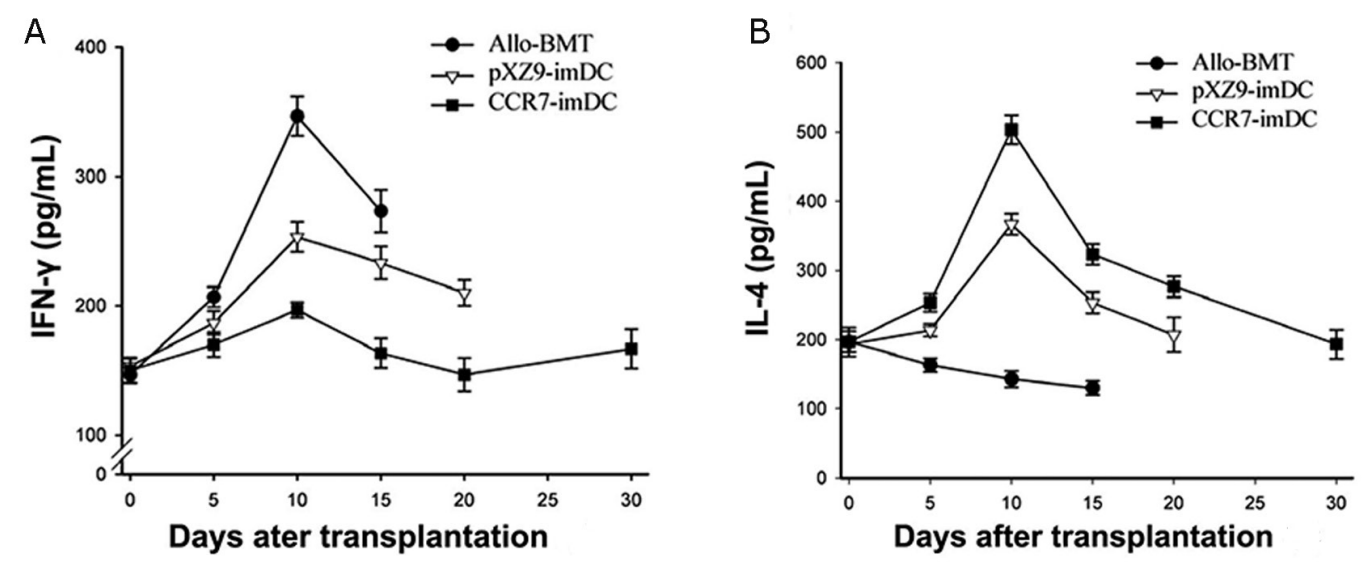

Figure 3. Post-bone marrow transplantation (BMT) plasma IFN- $\gamma$ (A) and IL-4 (B) levels in the different transplantation groups.

\section{Chimerism rate}

Bone marrow cells in recipient mice (in both pXZ9-imDC and CCR7-imDC groups) that had survived for longer than 30 days were collected and subjected to flow cytometry. The results showed that $\mathrm{H}-2 \mathrm{~K}^{\mathrm{b}}$-positive cells accounted for $95-100 \%$, which suggested complete chimerism, i.e., successful allo-BMT.

\section{DISCUSSION}

The ideal way to prevent GVHD after allo-HSCT and improve the success rate of transplantation lies in the establishment of transplantation tolerance between donor and 
recipient, which ensures that the immune cells of both maintain their normal immune function without mutual rejection. It has now been confirmed that DCs play a decisive role not only in the initiation of the immune response, but also in the induction of immune tolerance, depending on maturity. Immature DCs induce antigen-specific immune tolerance owing to their lack or low expression of MHC molecules and costimulatory molecules. This causes a reduction in the secondary signal necessary for $\mathrm{T}$ lymphocyte activation, resulting in inactivity, apoptosis, and low response in T cells. However, with high expression of both MHC molecules and costimulatory molecules, mature DCs activate the proliferation of lymphocytes, which are involved in the immune response. Therefore, the induction of immune tolerance by DC reconstruction has become a topic of great interest in research. The different phenotypes of DCs are decisive in the response of T lymphocyte upon antigen stimulation. CCR7 expressed in semi-mature DCs induces cell migration to the lymph nodes in the absence of danger stimulation signals, establishing peripheral immune tolerance targeting self-antigens (Jang et al., 2006; Seth et al., 2011). However, CCR7 is not naturally expressed in imDCs, so we attempted to modify imDCs with lentivirus vectors carrying the mouse $\mathrm{Ccr} 7$ gene (Xin et al., 2009), to improve their migration to secondary lymphoid organs and their immune tolerance effect. In the present study, the transfected imDCs maintained their original features while expressing high levels of CCR7 so that they were weak in stimulating allogeneic $T$ cell proliferation in vitro, but were significantly enhanced in their migration ability compared with unmodified imDCs under the effect of the CCR ligand CCL21. Our results, which are consistent with those of Riol-Blanco et al. (2005), justify further studies on transplantation tolerance.

In our acute GVHD models, the symptoms of the different mice appeared in more concentrated time spans, and the clinical manifestations and pathological changes of the target tissues were typical, which made them suitable for subsequent observation and follow-up studies on GVHD (Cao et al., 2010; Huang et al., 2010; Wang et al., 2012). In the present experiments, mice in the transplantation control group suffered from severe GVHD with high clinical scores. Although the severity of GVHD was alleviated and the survival prolonged in the empty vector group, mice that lived for more than 30 days accounted for only $10 \%$. In contrast, mice that had received an injection of CCR7-imDC showed significantly extenuated manifestations in both clinical symptoms and pathological changes. Additionally, survival was significantly prolonged and mice that lived for 30 days or longer reached about $40 \%$. These results suggest that genetically modified imDCs can effectively reduce the incidence and severity of GVHD in recipient mice, when compared with unmodified imDCs. This indicates a significantly improved ability of donorsourced imDCs to elicit immune tolerance after the transfection of LV-CCR7, which was probably through the interaction between CCR7 and its ligands (CCL19 and CCL21) in secondary lymphoid tissues. This interaction induced the chemotactic migration of imDCs to lymph nodes to present antigens to naive $\mathrm{T}$ lymphocytes, so that a signal of immune tolerance was passed on to remove previously formed autoantigen-specific $\mathrm{T}$ cells and to form new antigen-specific regulatory T cells (Barratt-Boyes and Thomson, 2005; Cools et al., 2008; Förster et al., 2008). This reversed the immune response targeting self-antigens and thus conferred peripheral immune tolerance on the recipient-derived antigens (the same auto- or self-antigens described above) through 'the re-domestication' of donor T cells. In addition, CCR7 also played a role in the regulation of DC structure, migration rate, maturity, and many other functions (Marsland et al., 2005; Förster et al., 2008). 
GVHD involves an extremely complex pathophysiological process. It is currently believed that the activation and amplification of donor $\mathrm{T}$ lymphocytes as well as the release of a large number of inflammatory cytokines are the two main factors involved in the occurrence of acute GVHD (Sun et al., 2007; Reddy et al., 2009). $\mathrm{CD}_{4}^{+} \mathrm{T}$ cells, especially Th cells, play an important role in the initiation and amplification of the severe GVHD pathological process. Th cells are classified into Th1 and Th2 cells according to their cytokine production and the active proteins they produce. Th1-dominant cells secrete the cytokines IL-2, IL-3, IFN- $\gamma$, and TNF, and are mainly characterized by IFN- $\gamma$, while Th2-dominant cells produce IL-4, IL-5, IL-6, IL-10, and IL-13, of which the secretion of IL-4 is the most prevalent. GVHD is likely to occur when the conversion of donor T cells from Th1 to Th2 is blocked during the initial response against host antigens (Zeis et al., 2001; Yi et al., 2009). In our study, the cytokine detection results showed that injections of CCR7-imDCs or pXZ9-imDCs both reduced the level of IFN- $\gamma$ (which was most likely to have been secreted by Th1 cells), and increased the amount of IL-4 (most probably produced by Th2 cells), suggesting that they might have induced donor $\mathrm{CD}_{4}^{+} \mathrm{T}$ cells to develop immune tolerance after they were activated by recipient-derived antigens. Th1 cytokines are involved in immune activation and cellular immune response, and can amplify the immunological effect by activating macrophages, assisting B cells, and promoting the proliferation of cytotoxic T cells. All the mechanisms described above participate in the promotion of GVHD. However, through the secretion of IL-4, Th2 cells can regulate the functions of Th1 cells and macrophages. In particular, Th2 cytokines can weaken the ability of Th1 cells to suppress cellular immune responses, and can thus participate in the regulation of immune tolerance and reduce the causative factors of GVHD (Sun et al., 2008). The secretion of cytokines with negative immunomodulatory effects by $\mathrm{Ccr} 7$ genemodified DCs and imDCs may be one of the mechanisms by which they induce relatively stable immune tolerance. However, the ability of transfected DCs to extenuate GVHD was quite limited, because about half of the recipient mice still died of GVHD eventually. The possible reasons for this may be as follows: 1) imDCs have the potential to mature, especially under the stimulation of viruses, such as the virus vectors used in our study, so that the imDCs injected in our study ultimately tended to become mature DCs; and 2) in the actual pathogenesis of GVHD, recipient APCs also play a certain role. Yet owing to the selective intervention of donor APCs in our study, there was no suppression of the recipient APCs, which probably caused the limited effect. Therefore, a combination including short-term immunosuppressants or injections at various time points may help achieve better results. We plan to carry out experiments addressing these issues in future studies.

In summary, our study has proved that by injecting modified imDCs after allo-HSCT, GVHD can be partially suppressed and the survival of the recipients can be prolonged, probably through the regulation of Thl/Th2-type cytokines. Our results have provided an experimental basis for the application of genetic engineering of imDCs in clinical practice regarding allo-HSCT. We believe that the in-depth study of genetically engineered DCs it will provide new ideas and treatment concepts for autoimmune-, tumor immunology-, and transplantation immunology-related issues, among others.

\section{Conflicts of interest}

The authors declare no conflict of interest. 


\section{ACKNOWLEDGMENTS}

Research supported by the Medical Scientific Research Foundation of Jiangsu Province (grant \#H 201427), the Xuzhou Science and Technology Plan Program (grant \#XM13B040), and The Ordinary College Postgraduates Practice and Innovation Projects of Jiangsu Province in 2013-2014 (grant \#CXLX13_99 and \#SJZZ_0200).

\section{REFERENCES}

Alpdogan O and van den Brink MR (2012). Immune tolerance and transplantation. Semin. Oncol. 39: 629-642.

Barratt-Boyes SM and Thomson AW (2005). Dendritic cells: tools and targets for transplant tolerance. Am. J. Transplant. 5: 2807-2813.

Blazar BR, Taylor PA, McElmurry R, Tian L, et al. (1998). Engraftment of severe combined immune deficient mice receiving allogeneic bone marrow via In utero or postnatal transfer. Blood 92: 3949-3959.

Cao J, Chen C, Zeng L, Li L, et al. (2010). Engineered regulatory T cells prevent graft-versus-host disease while sparing the graft-versus-leukemia effect after bone marrow transplantation. Leuk. Res. 34: 1374-1382.

Choi SW, Levine JE and Ferrara JL (2010). Pathogenesis and management of graft-versus-host disease. Immunol. Allergy Clin. North Am. 30: 75-101.

Cooke KR, Kobzik L, Martin TR, Brewer J, et al. (1996). An experimental model of idiopathic pneumonia syndrome after bone marrow transplantation: I. The roles of minor $\mathrm{H}$ antigens and endotoxin. Blood 88: 3230-3239.

Cools N, Ponsaerts P, Van Tendeloo VF and Berneman ZN (2007). Balancing between immunity and tolerance: an interplay between dendritic cells, regulatory T cells, and effector T cells. J. Leukoc. Biol. 82: 1365-1374.

Cools N, Van Tendeloo VF, Smits EL, Lenjou M, et al. (2008). Immunosuppression induced by immature dendritic cells is mediated by TGF-beta/IL-10 double-positive CD4+ regulatory T cells. J. Cell Mol. Med. 12: 690-700.

Edinger M, Powrie F and Chakraverty R (2009). Regulatory mechanisms in graft-versus-host responses. Biol. Blood Marrow Transplant. 15: 2-6.

Förster R, Davalos-Misslitz AC and Rot A (2008). CCR7 and its ligands: balancing immunity and tolerance. Nat. Rev. Immunol. 8: 362-371.

Hintzen G, Ohl L, del Rio ML, Rodriguez-Barbosa JI, et al. (2006). Induction of tolerance to innocuous inhaled antigen relies on a CCR7-dependent dendritic cell-mediated antigen transport to the bronchial lymph node. J. Immunol. 177: 7346-7354.

Hopkins RA and Connolly JE (2012). The specialized roles of immature and mature dendritic cells in antigen crosspresentation. Immunol. Res. 53: 91-107.

Huang Y, Feng S, Tang R, Du B, et al. (2010). Efficacy of pretreatment of allografts with methoxypolyethylene glycolsuccinimidyl-propionic acid ester in combination with an anti-OX40L monoclonal antibody in relieving graft-versushost disease in mice. Int J. Hematol. 92: 609-616.

Jang MH, Sougawa N, Tanaka T, Hirata T, et al. (2006). CCR7 is critically important for migration of dendritic cells in intestinal lamina propria to mesenteric lymph nodes. J. Immunol. 176: 803-810.

Jin Y, Fuller L, Ciancio G, Burke GW 3rd, et al. (2004). Antigen presentation and immune regulatory capacity of immature and mature-enriched antigen presenting (dendritic) cells derived from human bone marrow. Hum. Immunol. 65: 93-103.

Joo SY, Cho KA, Jung YJ, Kim HS, et al. (2010). Mesenchymal stromal cells inhibit graft-versus-host disease of mice in a dose-dependent manner. Cytotherapy 12: 361-370.

Kaplan DH, Anderson BE, McNiff JM, Jain D, et al. (2004). Target antigens determine graft-versus-host disease phenotype. J. Immunol. 173: 5467-5475.

Marsland BJ, Bättig P, Bauer M, Ruedl C, et al. (2005). CCL19 and CCL21 induce a potent proinflammatory differentiation program in licensed dendritic cells. Immunity 22: 493-505.

Morales-Tirado V, Luszczek W, van der Merwe M and Pillai A (2011). Regulatory immunotherapy in bone marrow transplantation. Sci. World J. 11: 2620-2634.

Pan MR, Hou MF, Chang HC and Hung WC (2008). Cyclooxygenase-2 up-regulates CCR7 via EP2/EP4 receptor signaling pathways to enhance lymphatic invasion of breast cancer cells. J. Biol. Chem. 283: 11155-11163.

Reddy P, Arora M, Guimond M and Mackall CL (2009). GVHD: a continuing barrier to the safety of allogeneic transplantation. Biol. Blood Marrow Transplant. 15: 162-168.

Riol-Blanco L, Sánchez-Sánchez N, Torres A, Tejedor A, et al. (2005). The chemokine receptor CCR7 activates in dendritic cells two signaling modules that independently regulate chemotaxis and migratory speed. J. Immunol. 174: 4070-4080. 
Seth S, Oberdörfer L, Hyde R, Hoff K, et al. (2011). CCR7 essentially contributes to the homing of plasmacytoid dendritic cells to lymph nodes under steady-state as well as inflammatory conditions. J. Immunol. 186: 3364-3372.

Sun K, Li M, Sayers TJ, Welniak LA, et al. (2008). Differential effects of donor T-cell cytokines on outcome with continuous bortezomib administration after allogeneic bone marrow transplantation. Blood 112: 1522-1529.

Sun Y, Tawara I, Toubai T and Reddy P (2007). Pathophysiology of acute graft-versus-host disease: recent advances. Transl. Res. 150: 197-214.

Travnik R, Beckers M, Wolff D, Holler E, et al. (2011). Graft-versus-host disease (GvHD) - an update. Part 2: prognosis and therapy of GvHD. Hautarzt. 62: 229-237.

Ueno H, Klechevsky E, Morita R, Aspord C, et al. (2007). Dendritic cell subsets in health and disease. Immunol. Rev. 219: 118-142.

Wang SH, Li DP, Zhang YJ, Zhang P, et al. (2012). Effects of immature dendritic cells genetically modified to express sTNFR I on graft-versus-host disease (GVHD) and graft-versus-leukemia (GVL) in allogeneic bone marrow transplantation mice. Zhonghua Xue Ye Xue Za Zhi. 33: 88-93.

Wilson NS, El-Sukkari D, Belz GT, Smith CM, et al. (2003). Most lymphoid organ dendritic cell types are phenotypically and functionally immature. Blood 102: 2187-2194.

Xin HM, Peng YZ, Yuan ZQ and Guo H (2009). In vitro maturation and migration of immature dendritic cells after chemokine receptor 7 transfection. Can. J. Microbiol. 55: 859-866.

Yi T, Chen Y, Wang L, Du G, et al. (2009). Reciprocal differentiation and tissue-specific pathogenesis of Th1, Th2, and Th17 cells in graft-versus-host disease. Blood 114: 3101-3112.

Zeis M, Uharek L, Hartung G, Glass B, et al. (2001). Graft-vs-leukemia activity and graft-vs-host disease induced by allogeneic Th1- and Th2-type CD4+ T cells in mice. Hematol. J. 2: 136-144.

Zhou F, Ciric B, Zhang GX and Rostami A (2013). Immune tolerance induced by intravenous transfer of immature dendritic cells via up-regulating numbers of suppressive IL-10(+) IFN- $\gamma(+)$-producing CD4(+) T cells. Immunol. Res. 56: 1-8. 